\title{
Effect of freezing on the passive mechanical properties of arterial samples
}

\author{
Jorge O. Virues Delgadillo ${ }^{1}$, Sebastien Delorme ${ }^{2}$, Rouwayda El-Ayoubi ${ }^{2}$, Robert DiRaddo ${ }^{2}$, Savvas G. \\ Hatzikiriakos ${ }^{1}$
}

\footnotetext{
${ }^{1}$ Department of Chemical \& Biological Engineering, University of British Columbia, Vancouver, Canada;

${ }^{2}$ Industrial Materials Institute, National Research Council of Canada, Boucherville, Canada.

Email: hatzikir@interchange.ubc.ca; Sebastien.Delorme@imi.cnrc-nrc.gc.ca
}

Received 19 December 2009; revised 18 January 2010; accepted 27 January 2010.

\begin{abstract}
Little mechanical data is available on human arteries because of the difficulty of testing artery samples often obtained from autopsy, while arteries are still considered "fresh". Various solutions mimicking the physiological environment have been used to preserve artery samples from harvesting to testing. Cryopreservation might provide a means to preserve the mechanical properties of arteries for days or weeks after harvesting. The objective of this study is to investigate the effect of several preservation methods, including simplified cryopreservation methods, on the passive mechanical properties of arteries. Eighteen fresh cruciform samples were mechanically tested. Samples were divided in three groups based on preservation medium and freezing method: isotonic saline solution, Krebs-Henseleit buffer solution with dimethyl sulfoxide (DMSO), and dipped in liquid nitrogen. In each group, half of the samples were stored at $-20^{\circ} \mathrm{C}$ and the other half at $-80^{\circ} \mathrm{C}$. Two months later, all the tissues were thawed at $4^{\circ} \mathrm{C}$ and mechanical tests were repeated. Preservation of arteries for two months in Krebs solution with DMSO (at $-20^{\circ} \mathrm{C}$ or at $-80^{\circ} \mathrm{C}$ ) or in isotonic saline solution at $-20^{\circ} \mathrm{C}$ were the methods that least changed the mechanical properties of the arteries.
\end{abstract}

Keywords: Thoracic Aorta; Mechanical Testing; Physiological Solution; Cryopreservation; Cryoprotective Agent

\section{INTRODUCTION}

The most common method for investigating the mechanical behavior of soft tissues consists of conducting mechanical tests on animal tissue explants, i.e., harvested within a day after the death of the animal [1-3]. There is however few data on in vitro mechanical behavior of human arterial tissues, in part because of the logistical difficulty of performing mechanical tests on fresh human tissues. Some studies [4-12] have reported testing human tissues several days after death, assuming that mechaniccal properties of the tissues were preserved by refrigerating and by using chemical solutions mimicking the physiological environment, such as saline, Ringer's, Tyrode's, Hank's, Krebs and variations based on these solutions. Cryopreservation methods [13] developed to preserve artery samples for re-implantation could also provide a means to preserve arteries for days and weeks before mechanical testing.

Artery mechanical properties have been shown to depend on the relative proportion and arrangement of the arterial wall constituents such as collagen, elastin and smooth muscle cells $[14,15]$, as well as on the integrity of the elastin and collagen fibers [16,17]. Smooth muscle cells contribute to structural, mechanical and functional changes in the arterial wall through several processes, including cell growth, elongation and reorganization of cells, and alteration of extracellular matrix composition [18]. The viability of smooth muscle cells, as well as the integrity of elastin and collagen fibers contribute to the arterial wall mechanical behaviour $[16,17]$. Damage caused to smooth muscle cells by ice formation and fragmentation of extracellular matrix fibers might affect the mechanical properties of the artery. Cryoprotective agents (CPA), such as dimethyl sulfoxide (DMSO), have been used in several studies to protect the cells from cryoinjury [19-21]. These CPAs are typically added to the storing solution in order to reduce ice formation in both intra- and extracellular space by preventing water movement out of the tissue [21]. The effect of cryopreservation has been investigated with respect to cell viability $[22,23]$ and histological changes [24-28], but little is known about the effect of cryopreservation on mechanical properties of the arterial wall.

It has been found $[29,30]$ that major arteries can withstand freezing and thawing without subsequent rupture. 
For instance, Pascual et al. [30] observed that slow thawing minimizes fissure and crack propagation compared to rapid thawing if the tissue was frozen at $-196^{\circ} \mathrm{C}$. They also observed that slow and rapid thawing does not significantly change the structure of the vessel frozen at $-80^{\circ} \mathrm{C}$. Fresh and cryopreserved behaviour of arteries has been investigated using inflation [31] and uniaxial tests [32,33]. Blondel et al., [31] and Venkatasubramanian et al., [33] observed significant stiffening of femoral arteries cryopreserved at $-80^{\circ} \mathrm{C}$ and $-150^{\circ} \mathrm{C}$ respectively compared to fresh arteries. Adham et al., [32] observed no difference in high strain modulus of aortas preserved at $+4^{\circ} \mathrm{C}$ for 1 month compared to cryopreservation with DMSO at $-135^{\circ} \mathrm{C}$ for 4 months.

The goal of the present study was to evaluate the effect of several conservation methods on the passive biaxial mechanical properties of arteries, regardless of cell viability, for the purpose of delayed mechanical testing. The preservation methods investigated include freezing for two months at either $-20^{\circ} \mathrm{C}$ or $-80^{\circ} \mathrm{C}$, in the presence of isotonic saline solution or Krebs-Henseleit solution with DMSO, or without immersing in solutions but dipped in liquid nitrogen. Because some of these methods are simplified adaptations of cryopreservation techniques, cell viability is not expected to be maintained in the samples. This study describes the storing conditions that best preserve the passive mechanical properties of arteries.

\section{MATERIALS AND METHODS}

\subsection{Experimental Setup}

Eighteen thoracic aortas were harvested within the day of death of pigs from a local slaughterhouse and cleaned of remaining connective tissue. One cruciform-shaped sample (55-by-55 mm) was cut out from each aorta for equibiaxial testing (Figure 1(a)). The average thickness of all tested specimens, measured with a vernier caliper, was $2.3 \pm 0.2 \mathrm{~mm}$. During transport and preparation, samples were stored in isotonic saline solution at $4^{\circ} \mathrm{C}$ for up to 8 hours prior to testing.

Cruciform samples were mechanically tested on a planar biaxial test bench (ElectroForce ${ }^{\circledR}$ LM1, Bose Corporation, Minnetonka, MN), shown in Figure 1(b) and capable of applying a peak force of $200 \mathrm{~N}$ over a displacement range of $12 \mathrm{~mm}$ per actuator. Samples were mounted in horizontal configuration inside a saline bath heated at body temperature $\left(37^{\circ} \mathrm{C}\right)$. Grip clamps were used to attach the four tabs of the cruciform sample to the arms extending from the actuators over the top of the bath, accordingly to a previously described method [34]. As well as being technically less difficult to set-up

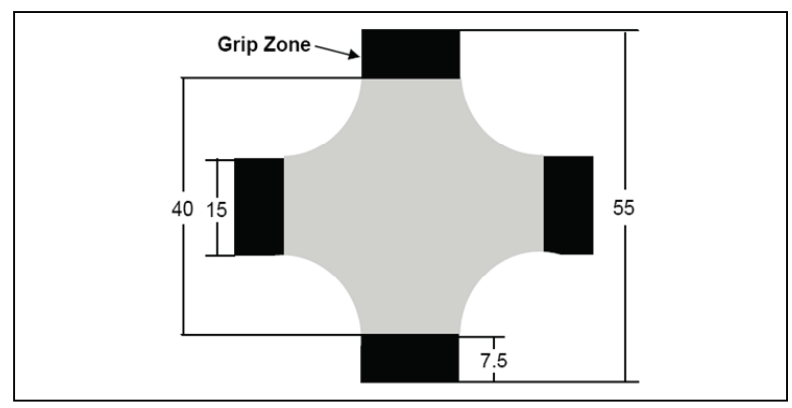

(a)

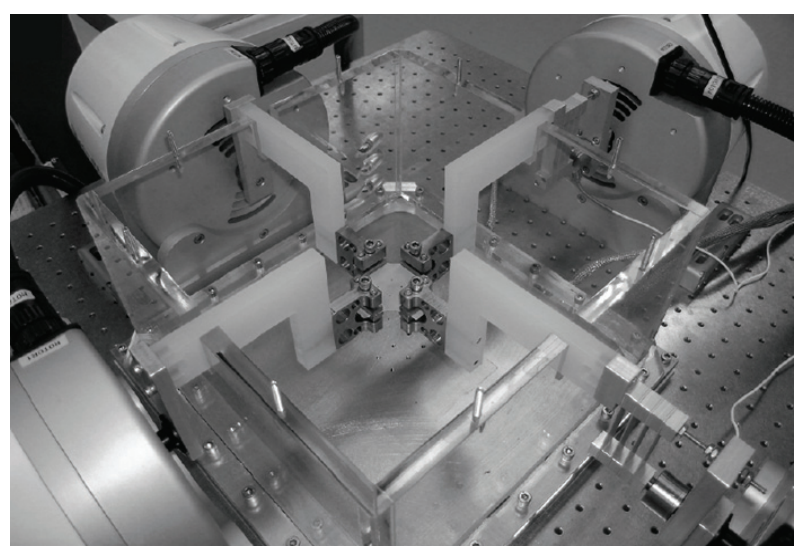

(b)

Figure 1. Sample dimension in millimetres for biaxial testing and (b) biaxial test bench used for cruciform sample clamped with grips.

than using square samples attached with hooks, and eliminating the need for videoextensometry, the cruciform sample method offers the advantage of stretching the samples to higher stretch ratios before failure than square samples with hooks [34]. The experimental data obtained from cruciform samples can be used in further studies (e.g. using inverse modeling) to obtain for example, which material parameters of the constitutive equation selected are most sensitive to freezing effects.

Equibiaxial testing was done by applying a $12 \mathrm{~mm}$ displacement (which corresponds to an average nominal stretch ratio of 1.55) on each one of the four grips, creating a non uniform strain distribution in the sample. The nominal stretch ratio was calculated using the distance between facing grips. The 1.55 stretch ratio was selected based on earlier experiments [34] because it allows capturing the nonlinear part of the stress-stretch curve, while avoiding rupture of the samples. Equibiaxial testing created a non uniform strain distribution in the cruciform sample. Triangular displacement wave forms were applied at a deformation rate of $110 \% / \mathrm{s}$, which corresponds to a frequency of $1.0 \mathrm{~Hz}$ (60 cycles per secod). Displacements were applied for 20 cycles. The first 10 
cycles were used for pre-conditioning. The force-stretch data was averaged over the last 10 cycles.

After the 18 fresh samples had been tested biaxially, they were randomized into three groups for storing: Group I, samples were put in a polypropylene tube filled with isotonic saline solution; Group II, samples were put in a polypropylene tube filled with Krebs-Henseleit solution, supplemented by $1.8 \mathrm{M}$ DMSO; and group III, samples were dipped in liquid nitrogen and then put in a polypropylene tube without any solution. For each group, half of the samples were stored at $-20^{\circ} \mathrm{C}$ and the other half at $-80^{\circ} \mathrm{C}$. Samples in group II were stored at $4^{\circ} \mathrm{C}$ for 20 minutes prior to freezing as proposed by Oliver [35]. Three samples were stored under each of the six different storage conditions, as shown in Table 1.

After two months, the samples were thawed at $4{ }^{\circ} \mathrm{C}$ for 24 hours and rinsed in isotonic saline solution at body temperature for one minute. The same mechanical testing procedure and conditions were then repeated.

\subsection{Statistical Analysis}

Medians and ranges (percentile between 75\%-25\%) were calculated for circumferential and axial force-stretch curves. Thawed/fresh force ratio $\left(F_{\max }^{\text {Thawed }} / F_{\max }^{\text {Fresh }}\right)$ at 1.55 stretch ratio were compared between all groups using the ANOVA Krustal-Wallis statistic test for independent variables with a 0.05 level of significance $(p=0.05)$. The $p$ value quantifies the probability of concluding that the storing condition used has an effect on the mechanical properties when in reality it did not. Krustal-Wallis test (non-parametric) was selected because the small sample size does not allow verifying the hypothesis of normal distribution, which is required to perform a Student's t-test. A statistical power analysis was also used to know the probability of falsely rejecting the null hypothesis using a threshold of $80 \%$.

\section{RESULTS}

\subsection{Experimental Results}

The medians of the force-stretch curves in the circumferential and axial directions for fresh specimens $(n=18)$ are plotted in Figure 2. The difference in mechanical behavior below a stretch ratio of 1.5 is small.

The medians of the force-stretch curves for the samples in group I, II and III are presented in Figures 3, 4 and 5 respectively. In groups I, II and III, preserved samples appeared to be stiffer than fresh samples in the circumferential direction, but these differences were not statistically significant. Preserved sample forces in the axial direction appeared to be similar to fresh samples.

Figures 6(a) and 6(b) shows the Thawed/fresh ratios $\left(F_{\max }^{\text {Thawed }} / F_{\max }^{\text {Fresh }}\right)$ of the circumferential forces measured at the maximum stretch ratio $(\lambda=1.55)$ for all the
Table 1. Number of samples for each storage condition.

\begin{tabular}{cccc}
\hline \multirow{2}{*}{ FreshTissue } & \multicolumn{3}{l}{ Thawed Tissue } \\
\cline { 2 - 4 } & Group & \multicolumn{2}{c}{ Storage Temperature } \\
\cline { 2 - 4 } & & $-20^{\circ} \mathrm{C}$ & $-80^{\circ} \mathrm{C}$ \\
\hline \multirow{2}{*}{18} & I & 3 & 3 \\
& II & 3 & 3 \\
& III & 3 & 3 \\
\hline
\end{tabular}

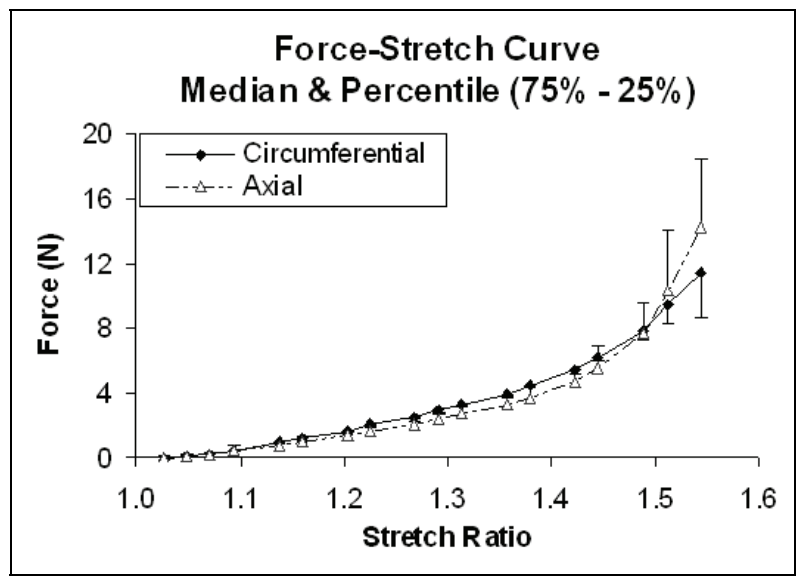

Figure 2. Medians of the force-stretch behavior of fresh cruciform samples tested at $110 \% / \mathrm{s}(n=18)$. Open triangles and closed circles represent axial and circumferential mean forces, respectively. The percentile of the data points is also shown.

samples stored at -20 and $-80^{\circ} \mathrm{C}$, respectively. The loading forces in the circumferential direction of all thawed samples (Groups, I, II and III) were almost two times higher than the loading forces of fresh samples (median of the ratio $F_{\max }^{\text {Thawed }} / F_{\max }^{\text {Fresh }} \cong 2.0$ ). On the other hand, difference in axial forces between fresh and thawed tissue was lower than $40 \%$ for all groups (Figures 6(c) and 6(d) shows axial results at -20 and $-80{ }^{\circ} \mathrm{C}$, respectively). The medians, percentiles (75\%-25\%) and the ANOVA Krustal-Wallis test $p$ values are also included in Figure 6.

\section{DISCUSSION}

This study investigated the influence of freezing in different solutions on the mechanical properties of arterial wall. Results were reported in terms of force vs. stretch ratio rather than stress vs. stretch ratio. This is due to the fact that stress and strain distributions in a cruciform sample subjected to biaxial extension experiment are not homogeneous, even when an equibiaxial stretch is applied. It was shown in [34] that the highest and lowest stresses can be found near the curved boundaries and near the center of the sample, respectively. 


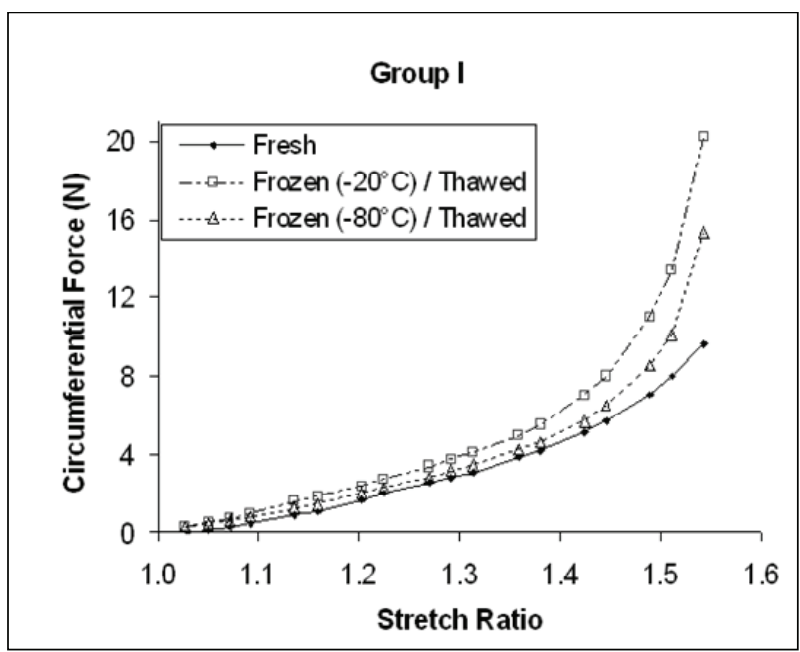

(a)

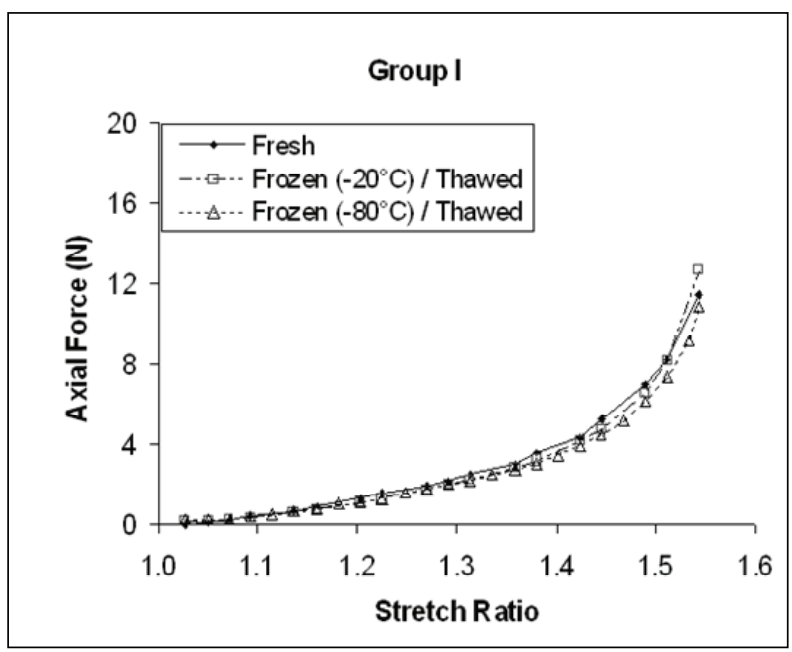

(b)

Figure 3. Medians of cruciform samples tested at a deformation rate of $110 \% / \mathrm{s}$, and stored in saline solution $(n=3)$ : Arterial wall behavior in (a) circumferential and (b) axial directions.

The differences observed in the mechanical behavior (i.e. medians of force data points) of thawed samples were not significant $(p=0.64)$, independently of the storing medium used (saline, Krebs with DMSO and dipping in liquid nitrogen). In order to confirm that indeed there is no differences in fresh and thawed behavior; a statistical power analysis (http://www.dssresearch.com/toolkit) was performed using the force data points obtained at 1.55 stretch ratio for each group between the fresh and the thawed specimens. The probability of rejecting a false null hypothesis (i.e. the storing condition used has no effect on the mechanical properties when in reality it might have an effect) and thus minimizing the occurrence of a $\beta$ error (which occurs if it is concluded that

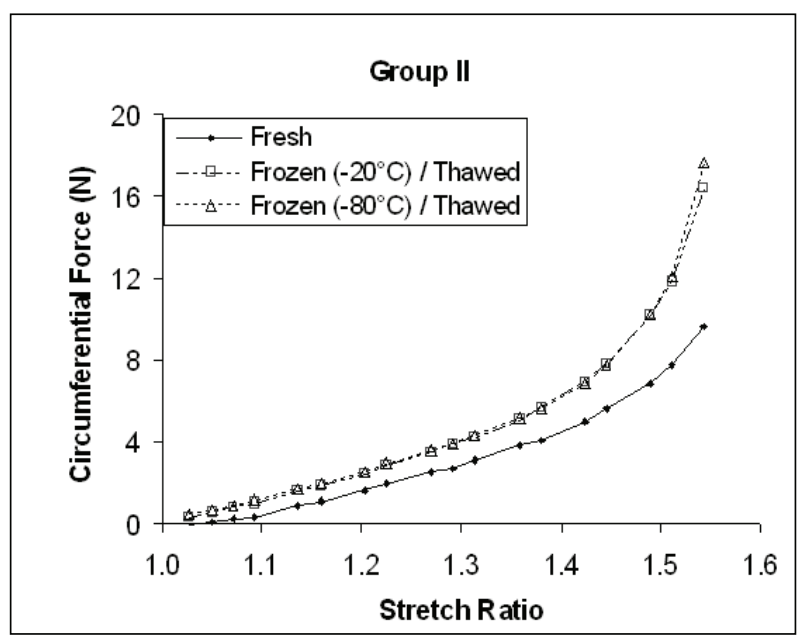

(a)

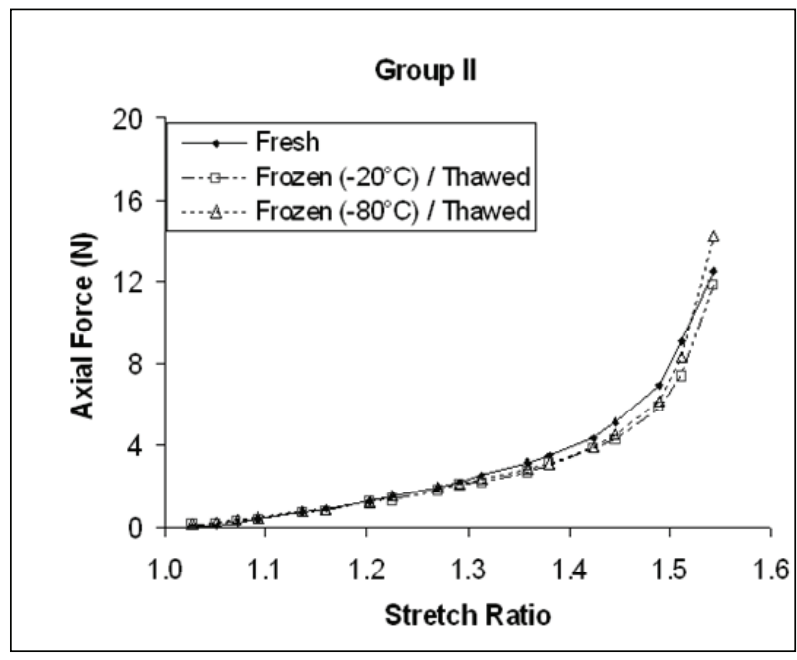

(b)

Figure 4. Medians of cruciform samples tested at a deformation rate of $110 \% / \mathrm{s}$, and stored in Krebs solution with dimethyl sulfoxide, DMSO ( $n=3$ ): Arterial wall behavior in (a) circumferential and (b) axial directions.

there is no difference in fresh and thawed specimen behavior when in reality there might be a difference) increases as the statistical power increases. Statistical powers between $13 \%$ and $93 \%$ were obtained in circumferential direction. In axial direction, the power was lower than $47 \%$ and higher than $5 \%$. The statistical power obtained for mostly all groups was not high enough to verify how significant the difference was between thawed and fresh tissue. In addition, the software used to obtain the power assumes that the data is parametric. Non-parametric analysis will likely estimate a slightly less power. Future studies with a higher sample size may further support the results obtained in this work. The observed lack of significant differences in this study was 


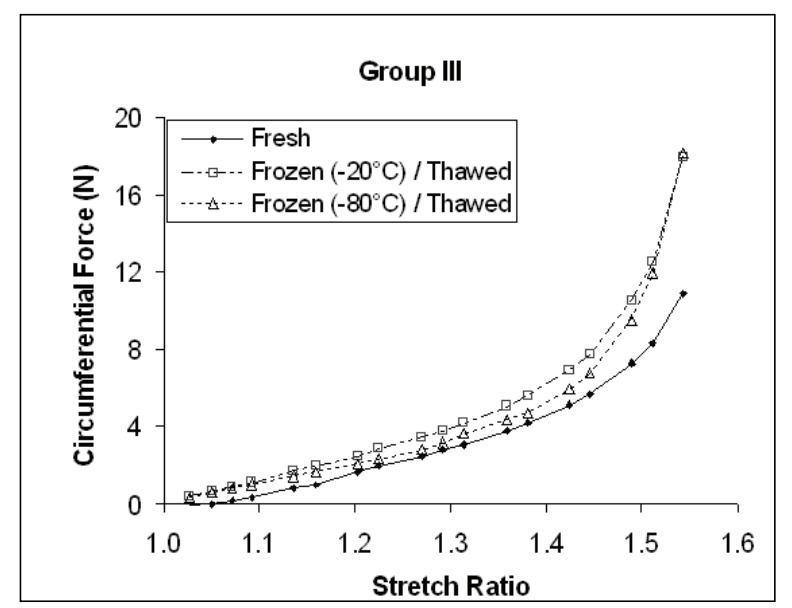

(a)

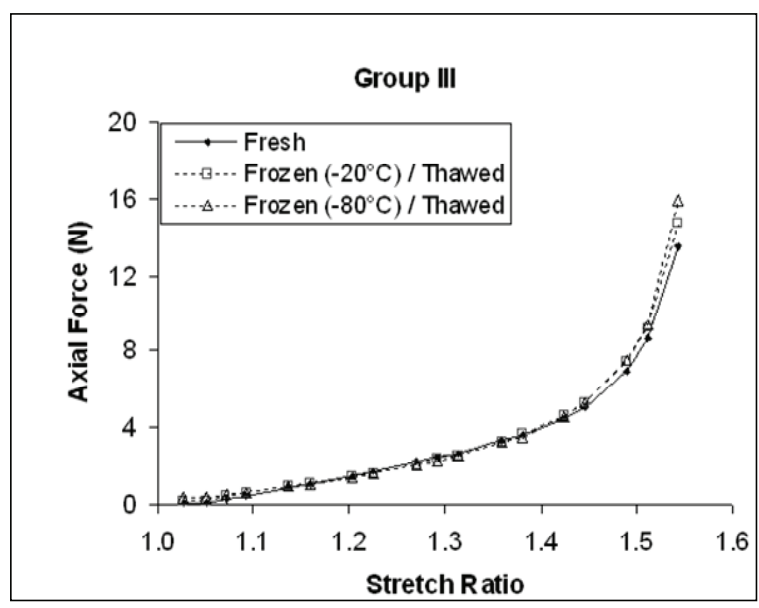

(b)

Figure 5. Medians of cruciform samples tested at a deformation rate of $110 \% / \mathrm{s}$, dipped in liquid nitrogen and stored in air $(n=3)$ : Arterial wall behavior in (a) circumferential and (b) axial directions.

likely due to the small number of samples tested per storing group $(n=3)$. However, these results are consistent with findings reported by Adham et al. [32] and by Venkatasubramanian et al. [33], who reported no significant difference between mechanical behavior of fresh artery samples and of samples cryopreserved with a cryoprotective agent such as DMSO, with up to 13 samples in each group.

The thawed samples appeared to be stiffer than the fresh samples at high stretch ratios. The differences in the load force-stretch curves might be the result of a modification in structure due to crosslinking or a change in fiber alignment. Elder et al. [36] stated that the increased tissue stiffness after cryopreservation may be related to the thermal change (i.e. drop of temperature) that occurs during preservation, catalyzing the thermal crosslinking of collagen fibers within the extracellular matrix. Collagen fibers are mainly oriented in the circumferential direction [37], thus the collagen matrix would develop stiffer interconnections in the circumferential than in axial direction during freezing. After thawing, the collagen matrix might have been reinforced in the circumferential direction, which is reflected in the stiffer response observed.

In this study the effect of freezing on cell injury and functionality was not investigated. However previous studies [38-40] have shown that contractile function of both endothelial and smooth muscle cells were preserved in arteries of a variety of species following freezing and thawing in DMSO solutions. DMSO reduces the mass transport of water and solutes throughout cell membrane while freezing. When water moves out of the cytoplasm, DMSO dissolves the suspended electrolytes, reducing the harmful effects of high solute concentration. DMSO interacts and partially replaces water molecules in such a way that the freezing point in the solution is lowered during cooling and the intracellular ice is reduced. Ice crystal formation and growth is prevented when the cryoprotectant-water mixture solidifies in a glass-like structure; and thus, preserving cell viability and mechanical properties. Song et al. [38] found that the maximum concentration needed to prevent damage in the tissue is $15 \%(\mathrm{wt} / \mathrm{wt})$. In the present study, the DMSO concentration was $1.8 \mathrm{M}$, i.e. $<12 \% \mathrm{wt} / \mathrm{wt}$. This concentration is expected to maintain cell viability within the tissue. Evidence has shown that after thawing, both biochemical and functional activities of arterial tissue cryopreserved at low temperatures in Krebs solution containing $1.8 \mathrm{M}$ of DMSO were comparable to fresh tissues [41].

Freezing and storing arteries in saline solution do not conserve the mechanical properties. As described by Lovelock [42], during the freezing process, the volume of liquid water in the cytoplasm decreases due to extracellular ice formation, leading to cellular dehydration. If the dehydration is too severe, the high electrolyte concentration inside the cytoplasm could result in cell death [43]. DMSO reduces the mass transport of water and solutes through the cell membrane while freezing. Ice crystal formation and growth is prevented when the cryoprotectant-water mixture solidifies in a glass-like structure, thus preserving cell viability and mechanical properties. The concentration of DMSO used in this study is expected to maintain cell viability within the tissue, albeit this study was not focused on freezing effects on cell viability. The better results obtained with DMSO suggest that cell death might play a role in changes of tissue stiffness, although this study could not unveil the exact mechanism involved. Also, during thaw- 


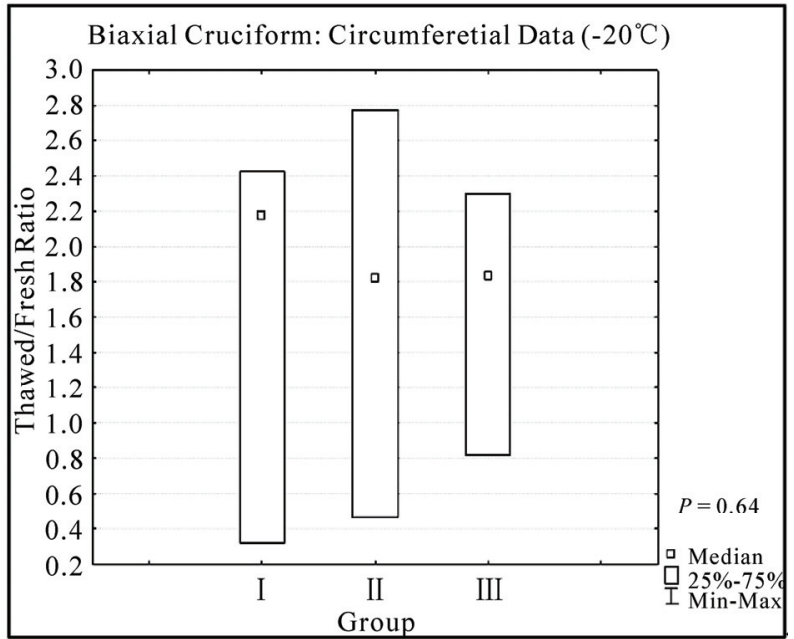

(a)

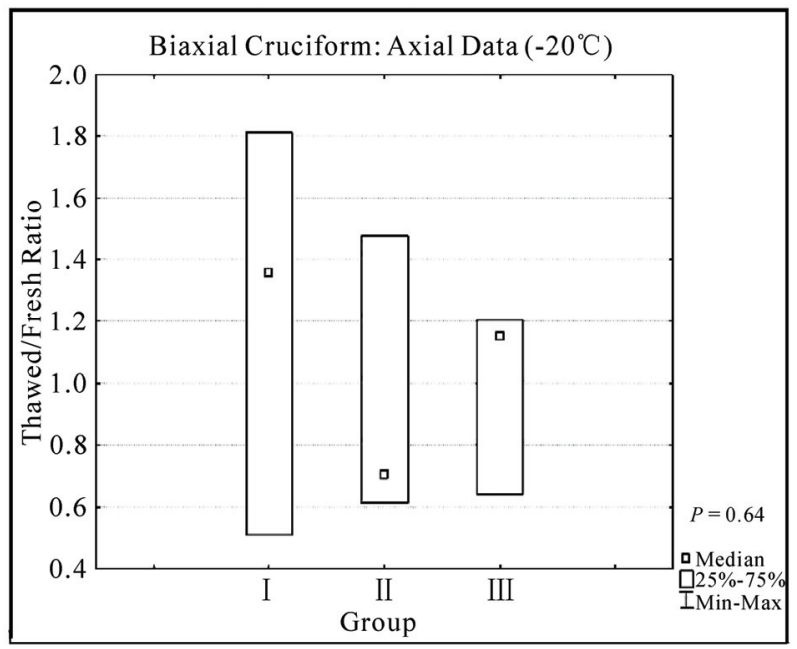

(c)

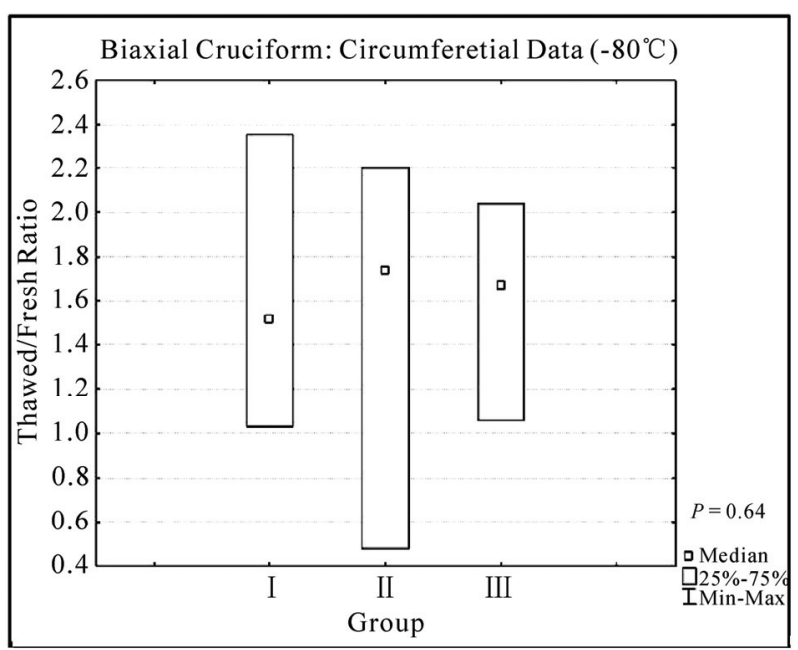

(b)

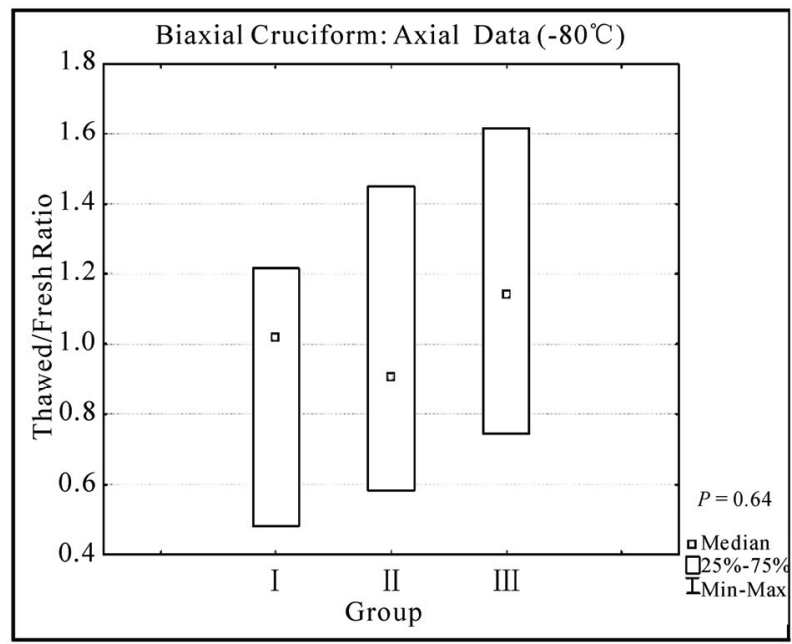

(d)

Figure 6. Comparison of Thawed/Fresh force ratios per storage group at the maximum stretch ratio applied $(\lambda=1.55)$ : $(a, b)$ circumferential and (c, d) axial direction $F_{\max }^{\text {Thawed }} / F_{\max }^{\text {Fresh }}$ ratios of samples stored at -20 and $-80^{\circ} \mathrm{C}$.

ing, the deformation of the cytoplasm due to volume increase could have permanently modified the orientation of the intracellular fibers, and as a result permanently changed the mechanical behaviour of the cells. Therefore our results suggest that, as concluded by Venkatasubramanian et al. [33], the changes in mechanical properties could be due to cell loss, to damage to the extracellular matrix or to a combination of both. Stachecki et al. [44] suggested that the reduction or removal of sodium from the storing solution is of primary importance to freeze cells efficiently, and proved that it is possible to replace sodium with a bigger molecular size ion, which encounters more obstacles to penetrate the cell membrane.

Dipping the arterial sample in liquid nitrogen could not preserve the mechanical properties of the specimens evaluated in this study. When exposing the specimens to a very rapidly decreasing temperature, ice crystal formation might have occurred faster than cell dehydratation, resulting in intracellular ice formation [45] and cell death. The differences observed between fresh and thawed samples suggest that the formation of intracellular ice crystals might have and effect on tissue stiffness. Vitrification is an alternative to obtain an amorphous glassy state matrix and thus minimizing ice nucleation and growth. However, it requires high concentrations of cryoprotective agents [46,47]. For example, Song et al. [48] were able to avoid crystallization by using a combination of CPAs (DMSO, formamide and 1,2-propanediol) and rapidly cooling the vessel to $-196^{\circ} \mathrm{C}$. Jiménez Rios and Rabin [49] have improved cell viability by using a specific freezing rate, pressurized liquid nitrogen, and specific type and concentration of CPA. Non-toxic cryopro- 
tectants, like glyerol [50] or fetal bovine serum [51] could also be used.

\section{CONCLUSIONS}

In the present study, we have found that the mechanical properties of arteries were not significantly changed after preserving arteries for two months in Krebs solution with DMSO (at $-20^{\circ} \mathrm{C}$ or at $-80^{\circ} \mathrm{C}$ ) or in isotonic saline solution at $-20^{\circ} \mathrm{C}$. However, the preservation approach taken here needs to be improved in order to maintain cell viability and as a result determine the best conditions to preserve the mechanical properties of the arterial wall. Selection of the optimal preservation method might be obtained by performing a study where the variables to be adjusted are slow cooling rate and step-wise DMSO loading, and therefore minimize changes to the arterial microstructure by the reduction of thermal stresses within the frozen tissue. Further studies are required to clarify the impact of cryopreservation on extracellular matrix architecture to help tailor an optimized approach in order to preserve the mechanical properties of arteries.

\section{ACKNOWLEDGEMENTS}

This research was possible thanks to the $\mathrm{PhD}$ scholarship given by the Mexican Council of Science and Technology (Consejo Nacional de Ciencia y Tecnología), CONACYT. The authors would also like to thank Marc-Andre Rainville for his guidance and assistance in sample preparation and mechanical testing.

\section{REFERENCES}

[1] Waldman, S.D. and Lee, J.M. (2002) Boundary conditions during biaxial testing of planar connective tissues: Part 1: Dynamic behavior. Journal of Materials Science, Materials in Medicine, 13(10), 933-938.

[2] Prendergast, P.J., Lally, C., Daly, S., Reid, A.J., Lee, T.C., Quinn, D. and Dolan, F. (2003) Analysis of prolapse in cardiovascular stents: A constitutive equation for vascular tissue and finite-element modelling. Journal of Biomechanical Engineering, 125(5), 692-699.

[3] Lally, C., Reid, A.J. and Prendergast, P.J. (2004) Elastic behavior of porcine coronary artery tissue under uniaxial and equibiaxial tension. Annals of Biomedical Engineering, 32(10), 1355-1364.

[4] Okamoto, R.J., Wagenseil, J.E., Delong, W.R., Peterson, S.J., Kouchoukos, N.T. and Sundt III, T. M. (2002) Mechanical properties of dilated human ascending aorta. Annals of Biomedical Engineering, 30(5), 624-635.

[5] Van dE Geest, J.P., Sacks, M.S. and Vorp, D.A. (2004) Age dependency of the biaxial biomechanical behavior of human abdominal aorta. Journal of Biomechanical Engineering, 126(6), 815-822.

[6] Mohan, D. and Melvin, J.W. (1983) Failure properties of passive human aortic tissue. II Biaxial tension tests. Journal of Biomechanics, 16(1), 31-44.

[7] Van Andel, C.J., Pistecky, P.V. and Borst, C. (2003) Me- chanical properties of porcine and human arteries: Implications for coronary anastomotic connectors. Annals of Thoracic Surgery, 76(1), 58-65.

[8] Schulze-Bauer, C.H.A.J., Mörth, C.H. and Holzapfel, G. A. (2003) Passive biaxial mechanics response of aged human iliac arteries. Journal of Biomechanical Engineering, 125(3), 395-406.

[9] Raghavan, M.L., Webster, M.W. and Vorp, D.A. (1996) Ex vivo biomechanical behavior of abdominal aortic aneurysm: assessment using a new mathematical model. Annals of Biomedical Engineering, 24(5), 573-582.

[10] Carmines, D.V., McElhaney, J.H. and Snack, R. (1991) A piece-wise non-linear elastic stress expression of human and pig coronary arteries tested in vitro. Journal of Biomechanics, 24(10), 899-906.

[11] Langewouters, G.J., Wesseling, K.H. and Goedhard, W.J. A. (1984) The static elastic properties of 45 human thoracic and 20 abdominal aortas in vitro and the parameters of a new model. Journal of Biomechanics, 17(6), 425435.

[12] Hudetz, G., Mark, G., Kovach, A.G.B., Kerenyi, T., Fody, L. and Monos, E. (1981) Biomechanical properties of normal and fibrosclerotic human cerebral arteries. Atherosclerosis, 39(3), 353-365.

[13] Han, B. and Bischof, J.C. (2004) Engineering challenges in tissue preservation. Cell Preservation Technology, 2(2), 91-112.

[14] Gamero, L.G., Armentano, R.L., Barra, J.G., Simon, A. and Levenson, J. (2001) Identification of arterial wall dynamics in conscious dogs. Experimental Physiology, 86(4), 519-528.

[15] Pascual, J.G., Garcia-Honduvilla, N., Gimeno, M.J., Jurado, F., Carrera-San, M.A. and Bellon, J.M. (2000) Rapid thawing increases the fragility of the cryopreserved arterial wall. European Journal of Vascular and Endovascular Surgery, 20(1), 13-20.

[16] Almassi, G.H., Farahbakhsh, B., Wooldribge, T., Rusch, N.J. and Olinger, G.N. (1996) Endothelium and vascular smooth muscle function in internal mammary artery after cryopreservation. Journal of Surgical Research, 60(2), 355-360.

[17] Pascual, G., Jurado, F., Rodríguez, M., Corrales, C., Lopez-Hervás, P., Bellón, J.M. and Bujan, J. (2002) The use of ischaemic vessels as prostheses or tissue engineering scaffolds after cryopreservation. European Journal of Vascular and Endovascular Surgery, 24(1), 23-30.

[18] Intengan, H.D. and Schiffrin, E.L. (2000) Structure and mechanical properties of resistance arteries in hypertension. Role of adhesion molecules and extracellular matrix determinants. Hypertension, 36(3), 312-318.

[19] Schenke-Layland, K., Madershahian, N., Riemann, I., Starcher, B., Halbhuber, K.J., König, K. and Stock, U.A. (2006) Impact of cryopreservation on extracellular matrix structures of heart valve leaflets. The Annals of Thoracic Surgery, 81(3), 918-926.

[20] Brockbank, K.G.M. (1989) Basic principles of viable tissue preservation. In: Clarke D.R., Ed., Transplantation techniques and use of cryopreserved allograft cardiac valves and vascular tissue. Adams Publishing Group, Boston, 9-23.

[21] Miles, C.A. (1999) Polymer-in-a-box mechanism for 
thermal stabilization of collagen molecules in fibers. Biophysics Journal, 76(6), 3243-3252.

[22] Devireddy, R.V., Neidert, M.R., Bischof, J.C. and Tranquillo, R.T. (2003) Cryopreservation of collagen-based tissue equivalents. I. Effect of freezing in the absence of cryoprotective agents. Tissue Engineering, 9(6), 1089-1100.

[23] Neidert, M.R., Devireddy, R.V., Tranquillo, R.T. and Bischof, J.C. (2004) Cryopreservation of collagen-based tissue equivalents. II. Improved freezing in the presence of cryoprotective agents. Tissue Engineering, 10(1/2), 23-31.

[24] Song, Y.C., Pegg, D.E. and Hunt, C.J. (1995) Cryopreservation of the common carotid artery of the rabbit: Optimization of dimethyl sulfoxide concentration and cooling rate. Cryobiology, 32(5), 405-421.

[25] Cui, X., Labarrere, C., He, L., Cheng, S., Siderys, H., Kovacs, R. and Gao, D. (2002) Cryopreservation and microsurgical implantation of rabbit carotid arteries. Cell Preservation Technology, 1(2), 121-128.

[26] Pacholewicz, J.K., Adkins, M.S., Boris, W.J., Gu, J., Xenachis, C., Klabunde, R.E., Jasionowski, T. and McGrath, L.B. (1996) Evaluation of cryopreserved internal thoracic artery as an alternative coronary graft: Evidence for preserved functional, metabolic and structural integrity. European Journal of Cardio-Thoracic Surgery, 10(1), 20-25.

[27] Hunt, C.J., Song, Y.C., Bateson, E.A.J. and Pegg, D.E. (1994) Fractures in cryopreserved arteries. Cryobiology, 31(5), 506-515.

[28] Wusteman, M.C. and Pegg, D.E. (2001) Differences in the requirements for cryopreservation of porcine aortic smooth muscle and endothelial cells. Tissue Engineering, 7(5), 507-518.

[29] Cooper, I.S., Samra, K. and Wisniewska, K. (1971) Effects of freezing on mayor arteries. Stroke, 2(5), 471-482.

[30] Pascual, G., García-Honduvilla, N., Rodríguez, M., Turégano, F., Bujan, J. and Bellón, M. (2001) Effect of the thawing process on cryopreserved arteries. Annals of Vascular Surgery, 15(6), 619-627.

[31] Blondel W.C.P.M., Lehalle, B., Maurice, G., Wang, X. and Stoltz, J.F. (2000) Rheological properties of fresh and cryopreserved human arteries tested in vitro. Rheologica Acta, 39(5), 461-468.

[32] Adham, M., Gournier, J.P., Favre, J.P., De La Roche, E., Ducerf, C., Baulieux, J., Barral, X. and Pouyet, M. (1996) Mechanical characteristics of fresh and frozen human descending thoracic aorta. Journal of Surgical Research, 64(1), 32-34.

[33] Venkatasubramanian, R.T., Grassl, E.D., Barocas, V.H., Lafontaine, D., Bischof, J.C. (2006) Effects of freezing and cryopreservation on the mechanical properties of arteries. Annals of Biomedical Engineering, 34(5), 823-832.

[34] Virues-Delgadillo, J. (2008) Mechanical properties of arterial wall. Ph.D. Dissertation, The University of British Columbia, Columbia.

[35] Olivier, S.A. and Ellington, J.E. (2003) Cryopreserving somatic cells with an arabinogalactan containing cryopreservation medium, US Patent, 6593138 B1.

[36] Elder, E., Chen, Z., Ensley, A., Nerem, R., Brockbank, K. and Song, Y. (2005) Enhanced tissue strength in cryo- preserved, collagen-based blood vessel constructs. Transplantation Proceedings, 37, 4525-4629.

[37] Humphrey, J.D. (1995) Mechanics of the arterial wall: Review and directions. Critical Reviews in Biomedical Engineering, 23(1-2), 1-162.

[38] Song, Y.C., Hunt, C.J. and Pegg, D.E. (1994) Cryopreservation of the common carotid artery of the rabbit. Cryobiology, 31, 317-329.

[39] Ku, D.D., Willis, W.L. and Caulfield, J.B. (1990) Retention of endothelium-dependent vasodilatory responses in canine coronary arteries following cryopreservation. Cryobiology, 27(5), 511-520.

[40] Bateson E.A.J. and Pegg, D.E. (1994) Cryopreservation of arteries: Selection of a model for human small elastic arteries and preliminary results of preservation of ringsegments with dimethyl sulphoxide. CryoLetters, 15(6), 15-26.

[41] Ellis, P. and Muller-Schweinitzer, E. (1991) Maintenance of functional activity of human pulmonary arteries after cryopreservation. British Journal of Pharmacology, 103(2), 1377-1380.

[42] Lovelock, J.E. (1953) The haemolysis of human red blood-cells by freezing and thawing. Biochimica et Biophysica Acta, 10(3), 414-426.

[43] Mazur, P., Rall, W.F. and Rigopoulos, N. (1981) Relative contributions of the fraction of unfrozen water and of salt concentration to the survival of slowly frozen human erythrocytes. Biophysical Journal, 36(3), 653-675.

[44] Stachecki, J.J., Cohen, J. and Willadsen, S. (1998) Detrimental effects of sodium during mouse oocyte cryopreservation. Biology of Reproduction, 59(2), pp. 395-400

[45] Toner, M., Cravalho, E.G. and Karel, M. (1990) Thermodynamics and kinetics of intracellular ice formation during freezing of biological cells. Journal of Applied Physics, 67(3), 1582-1593.

[46] Fahy, G.M., Macfarlane, D.R., Angell, C.A. and Meryman, H.T. (1984) Vitrification as an approach to cryopreservation. Cryobiology, 21(4), 407-426.

[47] Thakrar, R.R., Patel, V.P., Hamilton, G., Fuller, B.J. and Seifalian, A.M. (2006) Vitreous cryopreservation maintains the viscoelastic property of human vascular grafts. The FASEB Journal, 20(7), 874-881.

[48] Song Y.C., Khirabadi, B.S., Lightfoot, F., Brockbank, K. G.M. and Taylor, M.J. (2000) Vitreous cryopreservation maintains the function of vascular grafts. Nature Biotechnology, 18(3), 296-299.

[49] Jimenez Rios, J.L. and Rabin, Y. (2006) Termal expansion of blood vessels in low cryogenic temperatures Part I: A new experimental device. Cryobiology, 52(2), 269-283.

[50] Gao, D.Y., Lin, S., Watson, P.F. and Critser, J.K. (1995) Fracture phenomena in an isotonic salt solution during freezing and their elimination using glycerol. Cryobiology, 32(3), 270-284.

[51] Yiu, W.K., Cheng, S.W.K. and Sumpio, B.E. (2007) Direct comparison of endotelial cell and smooth muscle call response to supercooling and rewarming. Journal of Vascular Surgery, 46(3), 557-564. 